\title{
CORRECTION
}

\section{Correction to: Prediction of the sorption coefficient for the adsorption of PAHs on MWCNT based on hybrid QSPR-molecular docking approach}

\author{
Zahra Pahlavan Yali $^{1} \cdot$ Mohammad H. Fatemi $^{1}$
}

Published online: 21 March 2019

(c) Springer Science+Business Media, LLC, part of Springer Nature 2019

\section{Correction to: Adsorption}

https://doi.org/10.1007/s10450-018-9994-6

The original version of the article contained mistakes in equations. The corrected equations are given below.

$\mathrm{F}=\sum_{\mathrm{i}=0}^{\mathrm{i}=\mathrm{p}} \frac{\mathrm{y}_{\mathrm{i}}-\mathrm{y}_{0 \mathrm{i}}}{\mathrm{y}_{0 \mathrm{i}, \mathrm{Max}}-\mathrm{y}_{0 \mathrm{i}, \mathrm{Min}}}$

$\mathrm{VIF}=\frac{1}{1-\mathrm{R}^{2}}$

$\mathrm{Q}_{\mathrm{cv}}^{2}=1-\frac{\sum\left(\mathrm{y}_{\mathrm{i}}-\mathrm{y}_{0 \mathrm{i}}\right)^{2}}{\sum\left(\mathrm{y}_{0 \mathrm{i}}-\mathrm{y}_{\text {mean }}\right)^{2}}$

SPRESS $=\sqrt{\frac{\sum\left(\mathrm{y}_{\mathrm{i}}-\mathrm{y}_{0 \mathrm{i}}\right)^{2}}{\mathrm{n}-\mathrm{p}-1}}$

$\mathrm{ME}_{\mathrm{j}}=\frac{\beta_{\mathrm{j}} \sum_{\mathrm{i}=1}^{\mathrm{n}} \mathrm{d}_{\mathrm{ij}}}{\sum_{\mathrm{i}}^{\mathrm{m}} \beta_{\mathrm{j}} \sum_{\mathrm{i}}^{\mathrm{n}} \mathrm{d}_{\mathrm{ij}}}$

The original article has been corrected.

The original article can be found online at https://doi.org/10.1007/ s10450-018-9994-6.

Zahra Pahlavan Yali

zahrapahlavan64@gmail.com

1 Chemometrics Laboratory, Faculty of Chemistry, University of Mazandaran, Babolsar, Mazandaran, Iran 